\title{
Automatisierte Extraktion von Silbenquantitäten aus Digitalisaten lateinischer Texte
}

\author{
Robert Knapp \\ Universität Salzburg \\ robert.knapp@sbg.ac.at
}

\begin{abstract}
Die digitale Erfassung von Silbenquantitäten ist eine notwendige Vorstufe zu statistischen Untersuchungen größerer Corpora von lateinischer Kunstprosa und Dichtersprache. Wir diskutieren Möglichkeiten zur automatisierten Extraktion von Quantitätsinformationen aus einem Text und präsentieren einen lauffähigen Algorithmus. Dieser ist in Java implementiert. Das Programm nimmt im interaktiven Modus die Quantitätsanalyse selbständig vor, wo immer es möglich ist, überlässt aber in Zweifelsfällen dem Benutzer die Zuordnung.
\end{abstract}

\section{$1 \quad$ Einleitung}

Im Lateinischen gilt eine Silbe als lang, wenn sie einen langen Vokal oder einen Diphthong enthält (Naturlänge) oder wenn sie geschlossen ist (Positionslänge). Offene Silben mit kurzem Vokal gelten als kurz.

Die strikt geregelte Abfolge langer und kurzer Silben ist die konstituierende Eigenschaft antik-lateinischer Dichtung. In literarisch anspruchsvoller Kunstprosa begegnet anstelle der strengen Abfolge lediglich eine überzufällige statistische Häufung bestimmter Quantitätssequenzen in der Nähe inhaltlicher Einschnitte, die in der deutschsprachigen Forschung etwas irreführend als „Prosarhythmus“ bezeichnet wird (Aumont, 1996; Knapp, 2015).

Verwertbare Aussagen über dieses Phänomen bedürfen großer Stichproben und der Auswertung mit statistischen Methoden. Dazu müssen Quantitäten in Texten ausgezählt werden. Was in der Vergangenheit mit erheblichem Aufwand manuell geleistet wurde (Zielinski, 1904, 1914; de Groot, 1919a, 1919b, 1921; Primmer, 1968; Aili, 1979), sollte im Zeitalter des Computers in digitaler Form erfolgen, weil zeitgemäße statistische Verfahren rechenintensiv sind und daher am Computer durchgeführt werden.

Digitalisate antiker Texte liegen vor, beispielhaft sei die Perseus Digital Library genannt (Crane, o.J.). Sie enthalten aber meist nicht unmittelbar die Information, welche Silben lang und welche kurz sind.

Wir versuchen im Folgenden, genau diese Information automatisiert aus einem digitalen Text in lateinischer Sprache zu extrahieren.

\section{Ansätze und Stolpersteine auf dem Weg von der Wortform zur Silbenstruktur}

\subsection{Vokal: ja oder nein?}

Zunächst sind die silbentragenden Vokale zu identifizieren. Was einfach klingt - die lateinische Schrift unterscheidet die Vokalzeichen $\langle\mathrm{a}\rangle,\langle\mathrm{e}\rangle,\langle\mathrm{i}\rangle,\langle\mathrm{o}\rangle,\langle\mathrm{u}\rangle$ sowie $\langle\mathrm{y}\rangle$ in griechischen Fremdwörtern erweist sich im Fall von $\langle\mathrm{i}\rangle$ und $\langle\mathrm{u}\rangle$ als nicht trivial: Beide werden sowohl vokalisch (z.B. utilis) als auch halbvokalisch gebraucht (iam, ualde, quod).

Zwar unterscheiden sich die Verwendungen in ihrer Distribution (halbvokalisches $\langle i\rangle$ und $\langle u\rangle$ taucht nur vor Vokal auf, vokalisches bevorzugt vor Konsonant), doch sind die Details komplex, wie der Vergleich zwischen amaui (halbvokalisches $\langle\mathrm{u}\rangle$, vokalisches $\langle\mathrm{i}\rangle$ ), arcui (vokalisches $\langle\mathrm{u}\rangle$, vokalisches $<\mathrm{i}\rangle$ ) und alicui (vokalisches $\langle\mathrm{u}\rangle$, halbvokalisches $<\mathrm{i}>$ ) zeigt.

In einigen Fällen sind sogar beide Lesarten möglich, etwa bei wortfinalem -ia im Nom./Akk. Pl. n. bzw. Nom. Sg. f. (vgl. Verg. Aen. 1, 2 Laviniaque mit halbvokalischem <i> gegen Verg. Aen. 7, 72 Lavinia mit vokalischem $<\mathrm{i}>$ ). 
Konsequenz daraus ist, dass die Information, $o b<\mathrm{i}\rangle$ und $\langle\mathrm{u}\rangle$ vokalisch zu lesen sind oder nicht, nicht in allen Fällen aus dem Schriftbild ableitbar ist, sondern eine lexikalische Eigenschaft darstellt.

\subsection{Die klaren Fälle: Positionslängen und Diphthonge}

Geschlossene Silben gelten immer als lang, dies wird als Positionslänge bezeichnet. Im Schriftbild sind sie daran erkennbar, dass dem silbentragenden Vokal zwei oder mehr Konsonanten folgen. Dies gilt auch über Wortgrenzen hinweg.

Dass es von dieser Heuristik Ausnahmen gibt, ist bekannt, lässt sich aber vergleichsweise gut in eindeutige Regeln packen. Ein Beispiel ist die Regel Muta cum liquida: Lautkombinationen wie /br/ gehören phonologisch zur Folgesilbe und schließen die vorhergehende Silbe daher normalerweise nicht, es sei denn, eine Morphemgrenze liegt zwischen den beiden Elementen, so etwa in ab.rum.pe.re., oder auch gemäß literarischer Konvention.

Diphthonge $\langle a e\rangle,\langle o e\rangle,\langle u i\rangle,\langle e i\rangle,\langle a u\rangle,\langle e u\rangle$ enthalten einen Vokal mit nachfolgendem Halbvokal. Die Silbe, deren Tonträger ein Diphthong ist, ist immer lang. Nicht alle derartigen Digraphen sind jedoch als Diphthong zu lesen. Wir haben oben schon gesehen, dass <ui> auch zweisilbig sein kann (arcui). Auch griechische Fremdwörter sind reich an vokalischen Di- und Trigraphen, deren lautliche Realisierung nicht immer den lateinischen Regeln folgt.

Insgesamt sind aber Positionslängen und Diphthonge relativ verlässliche Ankerpunkte. Ein Algorithmus, dem keine lexikalischen Informationen vorliegen, hat sich primär auf diese beiden Phänomene zu stützen.

\subsection{Ohne Lexikon keine Chance: Naturlängen}

In offenen Silben entscheidet die Quantität des Vokals über die Silbenquantität. Vokalquantität ist im Lateinischen eine lexikalische Eigenschaft.

Leider zeigt die übliche Verschriftlichung die Vokallängen nicht an. Ein analysierender Algorithmus muss daher über ein eigenes Lexikon verfügen, dem die Vokalquantitäten entnommen werden.

Dazu wiederum ist notwendig, dass der Algorithmus die im Text auftretenden Wortformen seinen internen Lexikoneinträgen zuordnen kann.

Zwei Ansätze sind denkbar: Ein Wortformen-Lexikon enthält eine Liste von (graphischen) Wortformen. Es stellt geringe Ansprüche an den Algorithmus, ist aber dafür in Erstellung, Speicherung und Wartung sehr schwierig zu handhaben. In einer stark flektierenden Sprache wie dem Lateinischen gehören zu einem Lexem unter Umständen viele Dutzend mögliche Formen - man denke an Verbparadigmen. Enklitische Elemente wie -que, die im Prinzip an jede Wortform angehängt werden können und damit den gesamten Wortformen-Vorrat vervielfachen, sind dabei noch gar nicht berücksichtigt.

Das andere Extrem ist ein morphembasiertes Lexikon. Es ist wesentlich einfacher zu warten, verlangt aber dafür, dass der Algorithmus in der Lage ist, aus graphischen Wortformen die Morpheme herauszudestillieren.

In der Praxis liegt der richtige Weg in der Mitte. Nicht alle Morpheme, sondern gewisse lexikalische Basisformen werden im Lexikon gespeichert. Eine klare Struktur in der morphologischen Datenbank ermöglicht es dem Algorithmus, den überwiegenden Teil der möglichen lateinischen Wortformen korrekt zuzuordnen und mit Vokalquantitäten zu versehen.

\section{Morphologie und Lexikon}

\subsection{Ein Modell der lateinischen Morphologie}

Dem Algorithmus ist ein Modell der lateinischen Morphologie zugrundezulegen. Dazu ist ein Kompromiss zwischen Optimierung für die maschinelle Verarbeitung und intuitiver Verständlichkeit für den menschlichen Benutzer einzugehen. Das folgende Modell ist deshalb weder minimal im Sinne einer effizienten Verarbeitung noch linguistisch exakt im Sinne einer sprachhistorisch korrekten Beschreibung der lateinischen Sprache. Es stammt ursprünglich von Whitaker (2006) und wurde von Knapp (2015) für die Analyse von Vokalquantitäten adaptiert.

Jede Wortform wird als aus maximal vier Elementen zusammengesetzt angenommen. Das erste Element ist die Basis, der Träger der (lexikalischen) Semantik. Das zweite Element bezeichnen wir als Endung. Es ist Träger der grammatischen Information. 
Basen und Endungen tragen neben ihrer Semantik auch noch Informationen über die wechselseitige Kompatibilität. Zu diesem Zweck ist jede Basis und jede Endung einem Typ zugeordnet.

Das Typensystem orientiert sich an den klassischen „Flexionsklassen“ der lateinischen Grammatik, ist aber nicht in allen Details mit ihnen identisch.

Es ist hierarchisch und generisch strukturiert, sodass z.B. konsonantische Konjugation, i-Konjugation und Mischkonjugation mit ferre eine gemeinsame Oberklasse bilden und sich einen Großteil der Endungen teilen. Nur für die Formen, in denen sich die Klassen unterscheiden, gibt es spezifische Endungen für jede Unterklasse.

Die meisten Wortformen des Lateinischen lassen sich mit Basis und Endung bereits vollständig erfassen. Das dritte Element wird Basiserweiterung genannt. Es gehört semantisch zur Basis und wird für die Darstellung mancher Formen benötigt, weil es nicht vor, sondern nach der Flexionsendung steht (z.B. das Element -dam in quidam).

Als viertes Element gilt schließlich ein Enklitikon wie -que, das eine unabhängige Semantik trägt.

Einige Beispiele mögen verdeutlichen, wie das Modell konkrete Wortformen beschreibt:

- quo: Basis qu- (Klasse 1. Ebene „Pronomen“, Klasse 2. Ebene „qu-Pronomen“, Klasse 3. Ebene „qui/quae/quod“, Klasse 4. Ebene „Basis für Nom., Akk., Abl. Sg. sowie den gesamten Plural“), Endung -o („Abl. Sg. m./n.“, generisch für alle qu-Pronomina), keine Basiserweiterung, kein Enklitikon.

- cum: Basis cum- (Klasse „Präposition“), keine Endung, keine Basiserweiterung, kein Enklitikon.

- certamque: Basis cert- (Klasse 1. Ebene „Adjektiv“, Klasse 2. Ebene „a-o-Deklination“, Klasse 3. Ebene „us/a/um“), Endung -am („Nom. Sg. f.“, generisch für alle Adjektiva der a-o-Deklination), Enklitikon -que.

Einige wenige Wortformen sind in diesem Modell nicht beschreibbar. Sie erhalten einen Sonderstatus als Einzelformen. Ein Beispiel ist quisquis, in dem die Flexion an zwei Stellen auftaucht.

\subsection{Der Algorithmus zur morphologischen Analyse}

Der Algorithmus greift auf eine fünfteilige Datenbank zurück: Es gibt je eine Liste der Basen, Endungen, Basiserweiterungen, Enklitika und Einzelformen (Whitaker, 2006; Knapp, 2015).

Den Kern der Analyse bildet der Basis-Endungs-Algorithmus, dem ein Erkennungsprogramm für Einzelformen und Basiserweiterungen vorgeschaltet ist. Einzelformen werden zuallererst identifiziert, dann versucht das Programm Basiserweiterungen am Wortende abzutrennen und den verbleibenden Rumpf in die Basis-Endungs-Analyse einzuspeisen. Enklitika hingegen werden an dieser Stelle nicht gesucht.

Der Basis-Endungs-Algorithmus arbeitet mit einer Wortform aus $n$ Buchstaben. Basen und Endungen können auch aus einem leeren String bestehen (vgl. die Nullendung bei Präpositionen), daher ergeben sich $n+1$ mögliche Trennstellen. Jede Trennstelle liefert einen Ansatzpunkt für die Durchsuchung der Datenbanken. Beispiel $(n=5)$ :

$$
\begin{aligned}
& \text { dicam } \\
& \text { d.icam } \\
& \text { di.cam } \\
& \text { dic.am } \\
& \text { dica.m } \\
& \text { dicam. }
\end{aligned}
$$

Trennstellen, die entweder keine gültige Basis oder keine gültige Endung oder keine erlaubte Kombination aus beiden liefern, werden verworfen.

Eine kompatible Zusammenstellung aus Basis und Endung, die die gegebene Wortform abbildet, nennen wir im Folgenden eine Interpretation dieser Wortform. Das Programm stellt zu jeder Wortform eine Liste der Interpretationen zusammen.

Nur wenn bis zu dieser Stelle keine Interpretation gefunden worden ist, hält der Algorithmus nach Enklitika Ausschau. Dieses Verhalten musste eingeführt werden, um die Rate der Falsch-Positiv-Resultate für Enklitika zu senken.

Stimmt der Wortausgang mit einem Enklitikon aus der Liste überein, so wird dieses abgetrennt und der verbliebene Rumpf erneut in den Algorithmus eingespeist. 
Das Abbrechen des Algorithmus wäre selbst bei Vorhandensein eines Null-Enklitikons in der Datenbank gesichert, weil die Enklitikon-Suche je Wortform nicht mehr als einmal ausgeführt wird.

\subsection{Gewichtung}

Viele graphische Wortformen des Lateinischen haben mehr als eine Interpretation. In manchen Fällen sind die Interpretationen auch metrisch unterschiedlich, man denke an lëgis (2. Pers. Sg. Präs. Akt. Ind. von legere) vs. lègis (Gen. Sg. von lex).

Ein System von Gewichtungen berücksichtigt die Grundfrequenz der Lemmata im Wortschatz (Whitaker, 2006) und Frequenzmodulationen durch das Zusammenspiel von Basis und Endung (Knapp, 2015), um die Interpretationen nach ihrer Plausibilität zu ordnen. Dabei wird nach dem Grundsatz verfahren, dass Häufigeres wahrscheinlicher ist.

Die morphologischen Frequenzmodulationen berücksichtigen semantische Klassifikationen und weisen beispielsweise der Vokativform eines Abstraktums ein geringeres Gewicht zu als der Vokativform einer Personenbezeichnung. Weitere derartige Beziehungen mit verringertem Gewicht sind (Knapp, 2015, S. 64 f.):

- Lokativformen bei Nicht-Ortsbezeichnungen,

- aktive Formen von Deponentien,

- 1. und 2. Person bei unpersönlichen Verben,

- persönliches Passiv intransitiver Verben,

- Pluralwörter im Singular und Singularwörter im Plural.

Als dritte Ebene berücksichtigt der Algorithmus syntaktische Frequenzmodulationen. Beispielsweise triggert das Vorhandensein der Präposition cum die Erwartung für eine Ablativform in einem der nachfolgenden Wörter. Bei Wortformen, die direkt oder in geringer Entfernung nach cum stehen, begünstigt der Algorithmus deshalb die Interpretation als Ablativ relativ zu anderen Interpretationen.

Umgekehrt begünstigt das Vorhandensein einer Ablativform die Interpretation von cum als Präposition gegen die Interpretation als Subjunktion.

Weitere derartige syntaktische Regeln sind:

- Subjekt-Verb-Kongruenz (Nominativform und finites Verb im selben Numerus begünstigen einander, bei unterschiedlichem Numerus wirken sie gegeneinander),

- Kasusvalenz von Verben (Akkusativ ist in der Umgebung eines transitiven Verbs begünstigt),

- Substantiv-Adjektiv-Kongruenz (Substantiv und Adjektiv in übereinstimmendem Kasus, Numerus und Genus sind begünstigt),

- AcI-Konstruktion (Akkusativform und Infinitiv begünstigen einander).

Jede dieser Regeln ist intern durch zwei Parameter charakterisiert. Der erste beschreibt die Stärke der Heuristik (Kasusvalenz einer Präposition ist ein stärkerer Trigger als die Infinitiv-Erwartung in der Umgebung einer Akkusativform), der zweite ihre Fernwirkung (Kasusvalenz einer Präposition gilt nur in unmittelbarer Nähe, die Subjekt-Verb-Kongruenz erstreckt sich über den ganzen Satz).

\subsection{Algorithmus zur metrischen Analyse}

Jede morphologische Interpretation ist im zweiten Arbeitsschritt metrisch zu analysieren. Vokallängen und vokalische vs. konsonantische Lesarten können der Datenbank entnommen werden. Positionslängen sind berechenbar. Die Analyse erfolgt mehrfach iterativ, weil wortübergreifende Effekte (Positionslängen, Synaloephen) auf bereits analysierte Teile des Textes rückwirken.

In vielen Fällen kann auf diese Weise eine eindeutige Sequenz von Silbenlängen gewonnen werden.

Mehrdeutigkeiten ergeben sich durch Kann-Bestimmungen wie Muta cum liquida, durch trotz Gewichtungsheuristik unsichere morphologische Interpretationen und durch fehlende Daten.

Fehlende Daten können in zweierlei Gestalt auftreten: Entweder enthält die Datenbank zwar die graphischen Formen, sodass die morphologische Analyse gelingt, aber die metrischen Informationen sind nicht vorhanden, oder aber die Wortform konnte gar nicht erst analysiert werden.

Wenn entweder das Lemma oder seine Quantitäten nicht verfügbar sind, greift ein metrischer Basisalgorithmus, der anhand der Positionslängen alle formal zulässigen Silbenstrukturen ermittelt. 


\section{Implementierung}

\subsection{Interaktiver Modus}

Obigen Ausführungen ist zu entnehmen, dass die metrische Analyse zwar in vielen Fällen, aber bei weitem nicht immer eindeutig gelingt.

Die Entscheidung zwischen widerstreitenden morphologischen und/oder metrischen Interpretationen muss mitunter manuell erfolgen, wobei jedoch jede Änderung an einer Interpretation wegen der Gewichtungsregeln Rückwirkungen auf die Interpretationen in der Umgebung haben kann. Daher ist eine stetige Interaktion zwischen Programm und menschlichem Benutzer anzustreben.

Knapp $(2015 ; 2016)$ hat den in Abschnitt 3 skizzierten Algorithmus in einem Java-Programm Numerator mit graphischer Benutzeroberfläche implementiert.

Das Wörterbuch stammt in seiner Grundstruktur von Whitaker (2006), es wurde von Knapp (2015) im Zuge der praktischen Arbeit an Ciceros De natura deorum nach Bedarf ergänzt und umfasst aktuell rund 38000 Lemmata.

Die Quantitätsinformationen, bei Whitaker nicht vorhanden, hat Knapp (2015) mittels eines Hilfsprogramms aus dem Digitalisat von Lewis/Short (1879) importiert. Nur 21500 Einträge aus Lewis/Short konnten korrekt denen aus der Datenbank von Whitaker zugeordnet werden; bei rund 1000 hochfrequenten Einträgen hat Knapp die Quantitätsinformationen manuell ergänzt, sodass nun etwa 22500 Lemmata mit Quantitäten und 15500 weitere ohne Quantitäten zur Verfügung stehen (Knapp, 2015, S. A-30).

Im ersten Schritt führt Numerator den morphologischen und metrischen Algorithmus einmal vollständig aus. Farbige Markierungen zeigen dem Benutzer an, an welchen Stellen im Text Mehrdeutigkeiten und Unsicherheiten aufgetreten sind. Gelb steht für Wortformen, in denen keine metrische Interpretation mehr als 99\% der gesamten Gewichtungsmasse auf sich vereinigt, rot zeigt weniger als $80 \%$ an.

Ein Klick auf die Wortform öffnet im rechten Teil des Fensters eine Liste aller gefundenen Interpretationen, geordnet nach absteigendem Gewicht. Der Benutzer kann per Doppelklick eine davon auswählen oder noch spezifischer im rechten unteren Teilfenster eine metrische Interpretation zur gewählten morphologischen Interpretation wählen.

Falls die korrekte Interpretation nicht angeboten wird (z.B. bei nicht im Lexikon erfassten Lexemen oder im Fall von Fehlern in der Datenbank), kann der Benutzer wahlweise das Lexikon bearbeiten oder der Wortform manuell eine beliebige Quantitätenabfolge zuordnen.

Erfahrungsgemäß werden in einem Prosatext aus der Goldenen Latinität (prototypisch: Cicero) rund 90\% der Wortformen vollständig korrekt interpretiert. Je etwa 5\% fallen in den gelben und roten Bereich, wobei die gelb markierten Formen in den meisten Fällen nur durch einen Klick bestätigt werden müssen.

In Fachtexten und bei Häufung von Eigennamen, insbesondere griechischen, ist die Erfolgsquote des Algorithmus niedriger.

\subsection{Weiterverarbeitung}

Die gewonnenen Silbenstruktur-Sequenzen können anschließend in ein CSV-Format exportiert werden. Für die Analyse des Prosarhythmus sind insbesondere die Silbensequenzen in der Nähe inhaltlicher Einschnitte interessant. Zu diesem Zweck verfügt das Programm über eine rudimentäre Erkennungsfunktion für Einschnitte, die auf der Satzzeichensetzung im Input beruht. Auch diese Funktion ist, wie die übrigen Analysealgorithmen, interaktiv aufgebaut, sodass der Benutzer jederzeit an beliebigen Stellen im Text Einschnitte verschiedener Stärke setzen kann (vgl. die Ausführungen zur „Pausenstufe“ in Primmer, 1968).

Die Exportfunktion von Numerator ist auf diese Sinnabschnitte abgestimmt. Jeder Abschnitt wird zu einer Zeile der exportierten Datei. Zu jedem Abschnitt wird die Quantitätenabfolge in codierter Form ( 1 steht für lange Silben - syllaba longa -, b für kurze - syllaba brevis -, etc.), die Stärke des Einschnitts (Pausenstufe nach Primmer, 1968) sowie die Klauselform (Aili, 1979; Knapp, 2015) angegeben. 


\subsection{Versanalyse}

Als Nebenprodukt der Prosarhythmus-Funktionalität ergibt sich, dass die Software auch hervorragend zur metrischen Analyse lateinischer Verse geeignet ist.

In Versen ist die Quantitätenfolge streng geregelt, d.h. es gibt zu einem bestimmten Versmaß nur endlich viele Möglichkeiten, die ein einfach gestrickter Algorithmus durchlaufen und mit der zuvor ermittelten Silbenstruktur des Textes abgleichen kann.

Im Vers-Modus (Knapp, 2016) geht Numerator davon aus, dass jeder Zeilenumbruch das Ende eines Verses markiert, und verzichtet auf die Interpretation von Satzzeichen als Einschnitte. Der morphologische und metrische Algorithmus sowie der interaktive Modus sind gegenüber den obigen Ausführungen unverändert.

Die Versanalyse setzt auf den Ergebnissen auf. Der Benutzer kann per Kontrollkästchen diverse geläufige Versmaße auswählen, die in die Berechnung einbezogen werden sollen. Der Algorithmus durchläuft alle zulässigen Silbensequenzen der gewählten Versmaße und vergleicht sie zeilenweise mit dem Eingabetext.

Die Versanalyse kann grundsätzlich zwei Aufgaben bewältigen: Sie identifiziert aus einer beschränkten Auswahl, welches Versmaß vorliegt, und sie überlagert dem Text Betonungsstriche für ein iktierendes Lesen.

Ihre Trefferquote ist hoch, selbst wenn auf die interaktive Korrektur der gelb und rot markierten Wortformen verzichtet wurde. Fehler ergeben sich vor allem im Umgang mit griechischen Fremdwörtern und Eigennamen.

\subsection{Laufzeitkomplexität}

Die morphologische Analyse wird Wort für Wort abgearbeitet. In jedem $n$-buchstabigen Wort werden $n+1$ Datenbankabfragen in den Listen der Basen und Endungen durchgeführt. Enklitika und sonstige Besonderheiten sind demgegenüber quantitativ vernachlässigbar, die Anzahl der Datenbankabfragen im morphologischen Algorithmus wächst linear mit der Buchstabenanzahl des Textes.

Würde die metrische Analyse sogleich vollständig für alle gefundenen morphologischen Interpretationen ausgeführt, so ergäbe sich ein massives Laufzeitproblem: Bezeichne $r_{i}$ die Anzahl der gefundenen morphologischen Interpretationen zur i-ten Wortform. Um wortübergreifende Phänomene wie Positionslängen und Synaloephen vollständig zu erfassen, müsste der Algorithmus alle möglichen Zusammenstellungen der morphologischen Interpretationen zu allen Wörtern erfassen - davon gibt es $\prod_{i} r_{i}$ viele. Unabhängig von den tatsächlichen Werten der $r_{i}$ (realistische mittlere Werte von $r_{i}$ dürften zwischen 2 und 3 liegen), würde die Laufzeit jedenfalls exponentiell mit der Wortanzahl wachsen, längere Textpassagen wären so nicht analysierbar (Knapp, 2015).

Aus diesem Grund beschränkt sich die metrische Analyse darauf, wortübergreifende Phänomene nur für die derzeit aktive morphologische Interpretation zu berechnen, also für jene, die entweder vom Benutzer manuell ausgewählt ist oder das höchste Gewicht hat. So wird erreicht, dass auch die metrische Analyse nur von linearer Laufzeitkomplexität ist. Sie muss allerdings nach jeder Benutzeraktion neu berechnet werden.

Die Laufzeit des Versanalyse-Algorithmus wächst linear mit der Anzahl der Verse (Zeilen) und mit der Anzahl erlaubter Quantitätssequenzen. Die Quantität der letzten Silbe im Vers wird grundsätzlich nicht berücksichtigt. Unter dieser Voraussetzung hat der daktylische Hexameter als häufigstes Versmaß $2^{5}=32$ unterscheidbare Quantitätsmuster (jede der fünf ersten Senkungen kann entweder von einer langen oder von zwei kurzen Silben eingenommen werden). In den äolischen Versmaßen gibt es zumeist nur ein oder zwei mögliche Muster.

Im Kontrast dazu weist der jambische Senar $6^{5}=7776$, der jambische Oktonar sogar $6^{6}=46656$ unterscheidbare Muster auf, weil es für jeden nicht-finalen Jambus sechs verschiedene Realisierungen gibt: Die Senkung kann durch Länge, Kürze oder Doppelkürze, die Hebung durch Länge oder Doppelkürze ausgefüllt sein.

Die Analysezeit pro Zeile hängt somit stark davon ab, welche Versmaße berücksichtigt werden. Wenn die Komödienverse einbezogen sind, wächst die Laufzeit gegenüber einer ausschließlichen Berücksichtigung von Hexameter und äolischen Versen etwa um den Faktor 1000 - das kann auch auf aktuellen Geräten durchaus mehrere Minuten pro Zeile dauern. 


\section{$5 \quad$ Fazit}

Die metrische Analyse lateinischer Kunstprosa mit einem Computerprogramm ist möglich, sofern der metrischen Analyse eine morphologische Analyse vorausgeht, die mit einem Lexikon der Basisformen und ihrer Quantitäten gekoppelt ist.

Die Implementierung in Numerator (Knapp, 2015; 2016) zeigt, dass im interaktiven Modus produktives Arbeiten möglich ist, weil der Benutzer mit wenig Aufwand kritische Stellen im Text erkennen und dort manuell nachbessern kann. Auch längere Textpassagen können zusammenhängend bearbeitet werden, weil die Laufzeit der zentralen Analysealgorithmen lediglich linear mit der Wortanzahl wächst. Außerdem ergibt sich nebenher die Möglichkeit einer zuverlässigen Analyse quantitierender Versmaße. Automatische Erkennung des Versmaßes und iktierendes Lesen sind auf Knopfdruck verfügbar.

\section{Literatur}

Hans Aili (1979): The Prose Rhythm of Sallust and Livy. Acta Universitatis Stockholmiensis, Studia Latina Stockholmiensia XXIV, Stockholm.

Jacques Aumont (1996): Métrique et stylistique des clausules dans la prose latine. De Cicéron à Pline le Jeune et de César à Florus. Éditions Honoré Champion, vol. 56, Paris.

Gregory A. Crane (o.J., Hg.): Perseus Digital Library. Tufts University, Medford, Massachusetts, USA. Online: <http://www.perseus.tufts.edu>, Zugriff: 11.04.2018.

Albert Willem de Groot (1919a): De numero oratorio latino. Wolters, Groningen/Haag.

Albert Willem de Groot (1919b): A Handbook of Antique Prose-Rhythm. I: History of Greek Prose-Metre. Demosthenes, Plato, Philo, Plutarch and others. Wolters, Groningen/Haag.

Albert Willem de Groot (1921): Der antike Prosarhythmus. Zugleich Fortsetzung des Handbook of Antique ProseRhythm. Wolters, Groningen/Haag.

Robert Knapp (2015): Der Prosarhythmus als Stilmittel. Eine Untersuchung an Ciceros De natura deorum mit statistischer Auswertung und Softwareentwicklung. Diplomarbeit, Universität Salzburg.

Robert Knapp (2016): Numerator - die Software für lateinische Verse. Universität Salzburg. Online: <http://numerator.sbg.ac.at>, Zugriff: 11.04.2018.

Charlton T. Lewis und Charles Short (1879, Hg.): A Latin Dictionary. Founded on Andrew's edition of Freund's Latin dictionary. Clarendon Press, Oxford. Online als XML-Datei in der Perseus Digital Library (Crane, o.J.) verfügbar: <http://www.perseus.tufts.edu/hopper/ dltext?doc=Perseus\%3atext\%3a1999.04.0059>, Zugriff: 11.04.2018.

Adolf Primmer (1968): Cicero numerosus. Studien zum antiken Prosarhythmus. Österreichische Akademie der Wissenschaften, philosophisch-historische Klasse, Sitzungsberichte, 257. Band, Wien.

William Whitaker (2006): WORDS Version 1.97FC. Latin-English Dictionary Program. University of Notre Dame, Indiana, USA. Online unter <http://archives.nd.edu/whitaker/wordsdoc.htm>, Zugriff: 11.04.2018.

Thadeusz Zielinski (1904): Das Clauselgesetz in Ciceros Reden. Grundzüge einer oratorischen Rhythmik. SeparatAbdruck aus Philologus Supplementband IX, viertes Heft. Dieterich'sche Verlagsbuchhandlung, Leipzig.

Thadeusz Zielinski (1914): Der constructive Rhythmus in Ciceros Reden. Der oratorischen Rhythmik zweiter Teil. Dieterich'sche Verlagsbuchhandlung, Leipzig. 